\title{
ANÁLISE DO COMPORTAMENTO DO ORÇAMENTO E SUA EXECUÇÃO EM SAÚDE EM DIFERENTES GESTÕES DO MUNICÍPIO DE MONTES CLAROS NO PERÍODO DE 2005 A 2016
}

\author{
CASTRO, June Marize ${ }^{1}$ \\ VELOSO, Marcio Antônio Alves ${ }^{2}$ \\ MACEDO, Reginaldo Moraes de ${ }^{3}$ \\ SOARES, Simarly Maria ${ }^{4}$ \\ DUARTE, Simone Viana ${ }^{5}$
}

RESUMO: Este artigo aborda as despesas com a função saúde e suas sub funções no âmbito da administração pública municipal de Montes Claros/MG, durante três gestões. Foram consultados orçamentos de 2005 a 2016 nos registros da Secretaria Municipal de Saúde, atualizados pela inflação a partir da aplicação do Índice de Preços ao Consumidor Amplo(IPC-A). A série histórica foi analisada à luz da teoria e comparada com IBGE e Ministério da Saúde/. Os resultados principais apontam que o modelo de saúde percebido nas três gestões é hospitalocêntrico, corroborado pela baixa (taxa) cobertura do Programa Saúde da Família que está aquém do recomendado. Independente da gestão e da fonte de recurso, há certo padrão de gastos por sub função, sem descontinuidade do modelo assistencial. Mesmo havendo descontinuidades, não afetam gastos com saúde e cumprimento da Emenda Constitucional 29 (EC 29), o que pode significar que existe uma tendência de manutenção do gasto devido à estrutura de pactuação já consolidada.

Palavras-chaves: Saúde Pública. Gestão municipal. Orçamento. Despesas.

SUMMARY: This article deals with expenditures on the health function and its sub functions within the municipal public administration of Montes Claros / MG, during three administrations. Budgets from 2005 to 2016 were consulted in the records of the Municipal Health Department, updated by inflation from the application of the Extended Consumer Price Index (IPC-A). The historical series was analyzed in light of the theory and compared with IBGE and the Ministry of Health. The main results show that the health model perceived in the three administrations is hospital-centered, corroborated by the low (rate) coverage of the Family Health Program that falls short of what is recommended. Regardless of the management and the resource source, there is a certain pattern of expenditures by sub-function, without discontinuity of the care model. Even if there are discontinuities, they do not affect expenses with health and compliance with Constitutional Amendment (EC 29), which may mean that there is a tendency to maintain the expense due to the already consolidated agreement structure.

Keywords: Public Health. Municipal management. Budget. Expenses.

\section{INTRODUÇÃO}

A alocação e a disponibilidade dos recursos financeiros destinados à saúde são, certamente, fatores importantes para a operacionalização de um sistema municipal de saúde ou de outro qualquer programa ou projeto inserido nesse sistema. Para Couttolenc e Zucchi (2002), toda política ou conjunto de

\footnotetext{
${ }^{1}$ Doutoranda em Ciências da Informação pela Universidade Federal de Santa Catarinana. Professora da Universidade Estadual de Montes Claros.

${ }^{2}$ Professor da Universidade Estadual de Montes Claros.

${ }^{3}$ Doutor em Administração. Professor dos Departamentos de Ciências da Administração e da Computação da Unimontes e das Faculdades Santo Agostinho.

${ }^{4}$ Doutoranda em Administração pela Universidade de Brasília.Professora da Universidade Estadual de Montes Claros.

${ }^{5}$ Professora da Universidade Estadual de Montes Claros.
} 
prioridades se traduz num padrão específico de alocação de recursos, ainda que implícito. Diante da singularidade do espaço municipal, o conhecimento da alocação dos recursos financeiros nesse âmbito é importante para a identificação das dificuldades e facilidades na efetivação da descentralização e regionalização do Sistema Único de Saúde (SUS).

Nesse contexto, o orçamento público municipal é o planejamento da aplicação das receitas estimadas e das despesas previstas em custeios, investimentos, transferências e inversões, durante um período financeiro. E mesmo norteado por leis (Lei 4320/64; 101/00 e Constituição Federal de 1988), a gestão que faz a diferença é aquela que busca resultados, que beneficiem e modifiquem para melhor a realidade da sociedade local. Segundo Krusche e Ceretta (2012, p.1), “o orçamento público é um instrumento legal que deve integrar todo processo administrativo e financeiro direcionando a alocação dos recursos para as ações do Governo de forma planejada e integrada com a finalidade de atender as necessidades públicas".

Dessa forma, despesa pública pode ser definida como o conjunto de gastos realizados pelos entes públicos para custear os serviços públicos (despesas correntes) prestados à sociedade ou para a realização de investimentos (despesas de capital). Portanto, as despesas públicas devem ser autorizadas pelo Poder legislativo, através do ato administrativo chamado orçamento público.

Ao conhecer cada vez mais o espaço municipal onde se executam as ações de saúde e do seu papel na esfera local e regional na prestação de assistência à saúde, evidencia-se as peculiaridades do sistema de serviços de saúde de um município, pré-requisito para que se possa governá-lo com mais eficiência.

Tendo em vista a flagrante heterogeneidade das condições de vida e de atenção à saúde presentes nos diferentes recantos do território nacional, a necessidade de particularizar os achados dessa pesquisa se mostra de fundamental importância, sob pena de não se conseguir apreender essa diversidade de situações e, portanto, não se obter o conhecimento necessário para intervir adequadamente nos problemas e acompanhar o impacto das ações propostas. (COELHO; SZABZON; DIAS, 2014).

Nessa perspectiva, é relevante a discussão sobre o padrão de financiamento e de gasto em saúde do município em diferentes gestões de um município de porte médio com gestão plena, referência em região com características próprias, bem como o modelo de atenção à saúde sustentado financeiramente por esses recursos. Tal discussão é permeada pela análise da evolução da despesa pública com saúde, apresentada por subfunções ao longo da série histórica 2005 a 2016 do orçamento público.

Para isso, o objetivo geral foi analisar o comportamento das despesas por subfunção do orçamento municipal de Montes Claros, no período de 2005 a 2016, enquanto instrumento de planejamento para uma gestão pública eficaz.

Os objetivos específicos foram: comparar as despesas, continuidade e descontinuidades entre as administrações no período; verificar a alocação de recursos por subfunção e sua relação com o modelo de atenção à saúde sustentada; comparar despesas com receitas e levantar as taxas de coberturas de Estratégia de Saúde da Família no mesmo período.

\section{REFERENCIAL TEÓRICO}

A Constituição Federal definiu que "a saúde é direito de todos e dever do Estado" e a Lei Federal n. 8.080/1990, que regulamentou o SUS, prevê em seu Artigo $7^{\circ}$, como princípios do sistema, entre outros: I - universalidade de acesso aos serviços de saúde em todos os níveis de assistência; II - integralidade de assistência, entendida como conjunto articulado e contínuo das ações e dos serviços preventivos e curativos, individuais e coletivos, exigidos para cada caso, em todos os níveis de complexidade do sistema; [...]. 
A política de saúde, com vistas a um processo de mudança e melhora das condições de vida da população, deve vir sempre acompanhada de um contexto que apresente um modo de gestão dessa ação pública e seus respectivos monitoramentos e avaliação. Dessa forma, PENSAR o agir dos entes públicos é estabelecer, a partir das políticas, um constante processo de gestão que envolva o planejamento, o orçamento e seus respectivos controles e avaliações. Nessa perspectiva, é preciso ressaltar que os princípios e as diretrizes do SUS, previstos na Constituição Federal de 1988, asseguram uma base para a execução da política de saúde. Da mesma forma, o artigo $\mathrm{n}^{\circ} 165$ dessa Constituição estabelece os instrumentos do processo orçamentário público, o Plano Plurianual (PPA), a Lei de Diretrizes Orçamentárias (LDO) e a Lei Orçamentária Anual (LOA), que compreendem as metas e prioridades a serem executadas da administração pública, em geral, e da saúde, em particular (WEILLER; MENDES, 2016).

Os critérios de alocação de recursos para união, estados e municípios devem estar explícitos nos Planos de Saúde e legalmente registrados na Lei Orçamentária Anual (LOA), os quais são instrumentos de gestão em que os entes federados expressam as suas prioridades. Para Couttolenc e Zucchi (2002), o padrão de alocação de recursos pode revelar outra ordem de prioridades diferente daquelas expressas nos documentos formais das autoridades políticas ou sanitárias.

Assim, a alocação dos recursos financeiros registrada fortalece ou não o discurso municipal, a ampliação do acesso à Atenção Básica através do Programa de Saúde da Família significando estratégia para implantar a mudança do modelo de atenção, por exemplo, ou detecta-se a centralização no atendimento hospitalar, curativo, fragmentado e de baixa resolutividade ou a presença de núcleos de promoção à saúde e à assistência.

Além disso, a Emenda Constitucional no 29 (EC 29), aprovada em agosto de 2000 e regulamentada em 2012(LEI COMPLEMENTAR N ${ }^{\circ}$ 141, DE 13 DE JANEIRO DE 2012), estabeleceu que estados e municípios devem alocar, no primeiro ano, pelo menos, $7 \%$ dessas receitas, sendo que esse percentual deve crescer anualmente até atingir, para os estados, 12\%, no mínimo, em 2004 e, para os municípios, 15\% no mínimo. Em relação à União, a EC 29 determina que, para o primeiro ano, deveria ser aplicado o aporte de pelo menos 5\% em relação ao orçamento empenhado do período anterior; para os seguintes, o valor apurado no ano anterior é corrigido pela variação do Produto Interno Bruto (PIB) nominal.

Em abril de 2006, com a publicação da Portaria GM/MS nº. 699 aconteceu a regulamentação das Diretrizes Operacionais dos Pactos pela Vida e de Gestão e a instituição de uma nova forma de transferência de recursos federais destinados ao custeio de ações e serviços de saúde em blocos de financiamento, com a publicação, também, da portaria GM/MS nº. 698(5-6).

Essas diretrizes vêm salientar a importância da regionalização e de instrumentos de gestão como o Plano Diretor de Regionalização (PDR), Plano Diretor de Investimento (PDI) e a Programação Pactuada e Integrada (PPI). De fato, são todos instrumentos que possibilitam a reorganização dos processos de gestão e de regulação do sistema de saúde, buscando a melhoria e qualificação do acesso do usuário do SUS.

As responsabilidades com a gestão e o financiamento do SUS são compartilhadas entre os seus gestores dos três âmbitos. Segundo o Conselho Nacional de Saúde (2006), o Pacto pela Saúde é um conjunto de reformas institucionais do SUS pactuado entre as três esferas de gestão (União, Estados e Municípios) com o objetivo de promover inovações nos processos e instrumentos de gestão, visando alcançar maior eficiência e qualidade das respostas do Sistema Único de Saúde. Ao mesmo tempo, o Pacto pela Saúde redefine as responsabilidades de cada gestor em função das necessidades de saúde da população e na busca da equidade social. 
Por isso, a implementação do Pacto pela Saúde se dá pela adesão de Municípios, Estados e União ao Termo de Compromisso de Gestão (TCG). Então, o TCG substitui os processos de habilitação das várias formas de gestão anteriormente vigentes e estabelece metas e compromissos para cada ente da federação, sendo renovado anualmente.

Segundo Chioro (2013)², o papel do município na Gestão da Saúde é: (a) contratação, controle, auditoria e pagamento aos prestadores de serviços ambulatoriais e hospitalares; (b) administração da oferta de procedimentos ambulatoriais de alto custo e alta complexidade; (c) operação dos sistemas de informações do SUS; (d) avaliação do impacto das ações do Sistema de Saúde sobre as condições de vida e saúde; (e) execução das ações de vigilância à saúde (sanitária, epidemiológica, zoonoses, controle de endemias, dentre outros); e (f) gestão dos servidores da saúde, próprios e municipalizados.

Diante disso, a relação com os municípios tem como espaço de negociação e decisão política, a Comissão Intergestores Bipartite (CIB), em que as decisões devem ser tomadas por consenso. Na verdade, é recomendável que o próprio Secretário Estadual de Saúde participe da coordenação da Comissão Bipartite, já que se trata de um espaço político e assim as decisões sobre a condução do processo de implantação do SUS e, inclusive quanto à alocação dos recursos federais, são tomadas por essa Comissão.

Já no que diz respeito à participação da comunidade, a mesma se concretiza por meio de Conferências de Saúde e pelos Conselhos de Saúde. Essas duas instâncias foram instituídas em cada esfera de governo pela Lei 8.142/90 que, além de dispor sobre a participação da comunidade na gestão do SUS, trata das transferências intergovernamentais de recursos financeiros na área da saúde. Nesse âmbito, as Conferências de Saúde são realizadas com periodicidade quadrienal, com representantes dos vários segmentos sociais, com o objetivo de avaliar a situação de saúde e propor as diretrizes para a formulação das políticas de saúde nos níveis correspondentes. Essas Conferências se realizam em um processo ascendente desde Conferências Municipais de Saúde, passando por uma Conferência Estadual de Saúde em cada Estado e culminando em uma Conferência Nacional de Saúde.

Logo, o pacto pela saúde tem como pressupostos teóricos alguns conceitos relacionados à construção de rede de assistência por linha de cuidado, baseada na economia de escala e escopo e redes e Pacto Federativo (MENDES, 2007).

Nesse aspecto, Mendes (2002), quando fala em redes refere-se às redes de atenção, que têm três funções: "a função de resolução, a função de coordenação e a função de responsabilização". Assim, a função de resolução, intrínseca à sua instrumentalidade como ponto de atenção à saúde, consiste em solucionar a grande e maioria dos problemas de saúde; a função de coordenação, relacionada ao desempenho do papel de centro de comunicação, consiste em organizar os fluxos e contra-fluxos das pessoas e coisas pelos diversos pontos de atenção à saúde da rede; e a função de responsabilização consiste em co-responsabilizar-se pela saúde dos usuários em quaisquer pontos de atenção em que estejam sendo atendidos".

Da mesma forma, as formas de transferência dos recursos federais para estados e municípios também foram modificadas pelo Pacto pela Saúde, passando a ser integradas em cinco grandes blocos de financiamento ou subfunções (Atenção Básica, Média e Alta Complexidade da Assistência, Vigilância em Saúde, Assistência Farmacêutica e Gestão do SUS). (PORTARIA MOG no 42, de 14 de abril de 1999).

Desse modo, a função pode ser traduzida como o maior nível de agregação das diversas áreas de atuação do setor público. De fato, a função se relaciona com a missão institucional do órgão, por exemplo, cultura, educação, saúde, defesa. Já a subfunção representa um nível de agregação imediatamente inferior à função e deve evidenciar cada área da atuação governamental, por intermédio da agregação de determinado subconjunto de despesas e identificação da natureza básica das ações que se organizam

${ }^{2}$ Secretário de Saúde de São Bernardo do Campo e Presidente do Cosems-SP, 2013.

Nucleus,v.16,n.2,out.2019 
organizam em torno das funções, segundo o CONASS (2007):

Nesse cenário, a subfunção Atenção básica é um conjunto de ações, realizadas de maneira individual ou mesmo coletivamente e que ocorrem no primeiro nível de atenção dos sistemas de saúde, com o intuito da promoção, prevenção tratamento e reabilitação da saúde (PORTARIA nº 648/GM de 28 de março de 2006), ou seja, consolida e qualifica a Estratégia da Saúde da Família como modelo de atenção básica à saúde e como centro ordenador das redes de atenção à saúde do SUS. Portanto, a atenção básica é entendida como o primeiro nível da atenção à saúde no SUS (contato preferencial dos usuários), que se orienta por todos os princípios do sistema, inclusive a integralidade, mas emprega tecnologia de baixa densidade.

Em relação à infraestrutura necessária para a atenção básica, destaca-se a unidade básica de saúde, com ou sem saúde da família, com equipe multiprofissional composta por médico, enfermeiro, cirurgião dentista, auxiliar de consultório dentário ou técnico em higiene dental, auxiliar de enfermagem ou técnico de enfermagem e agente comunitário de saúde, entre outros.

Ao mesmo tempo, a assistência de Média complexidade ambulatorial é composta por ações e serviços que visam atender aos principais problemas e agravos de saúde da população, cuja complexidade da assistência na prática clínica demande a disponibilidade de profissionais especializados e a utilização de recursos tecnológicos, para o apoio diagnóstico e tratamento.

Já a alta complexidade é um conjunto de procedimentos que, no contexto do SUS, envolve alta tecnologia e alto custo, objetivando propiciar à população acesso a serviços qualificados, integrando-os aos demais níveis de atenção à saúde (atenção básica e de média complexidade).

Parta tal propósito, a Política Nacional de Atenção Hospitalar (PNHOSP) no âmbito do SUS foi

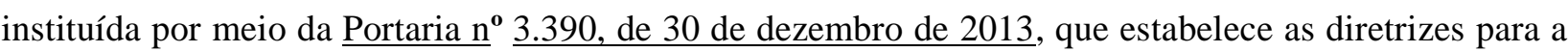
organização do componente hospitalar da Rede de Atenção à Saúde (RAS).

Art. $3^{\circ}$ Os hospitais são instituição complexas, com densidade tecnológica especifica, de caráter multiprofissional e interdisciplinar, responsável pela assistência aos usuários com condições agudas ou crônicas, que apresentem potencial de instabilização e de complicações de seu estado de saúde, exigindo-se assistência contínua em regime de internação e ações que abrangem a promoção da saúde, a prevenção de agravos, o diagnóstico, o tratamento e a reabilitação.

A subfunção Vigilância em Saúde é composta por dois componentes: da Vigilância Epidemiológica e Ambiental em Saúde e o componente da Vigilância Sanitária em Saúde. Ela envolve as ações de prevenção e controle de doenças transmissíveis, verificação de fatores de risco para o desenvolvimento de doenças crônicas não transmissíveis, saúde ambiental e do trabalhador para a análise de situação de saúde da população brasileira.

A Assistência Farmacêutica abrange três componentes: básico da assistência farmacêutica; estratégico da assistência farmacêutica; medicamentos de dispensação excepcional.

Nessa conjuntura, a Gestão do SUS consiste em pactuar de forma tripartite, aspectos relacionados a Descentralização; Regionalização; Financiamento; Programação Pactuada e Integrada; Regulação; Participação e Controle Social; Planejamento; Gestão do Trabalho e Educação na Saúde.

Ademais, recentemente essa legislação foi modificada por meio da Portaria $n^{\circ} 3.992$, de 28/12/2017. Seu conteúdo trata do financiamento e da transferência dos recursos federais para as ações e os serviços públicos de saúde do Sistema Único de Saúde (SUS), alterando a Portaria de Consolidação n 6/GM/MS de 28/09/2017, que contemplava a portaria $n^{\circ}$ 204/2007. As principais mudanças contempladas na nova portaria são as seguintes: (a) a forma de transferência dos recursos financeiros para custeio e investimento, uma vez que os recursos para custeio serão transferidos para uma só conta corrente no bloco 
de custeio e os recursos para investimentos ainda não contemplados com repasse serão transferidos para uma só conta corrente no bloco de investimento; (b) a junção dos antigos blocos de financiamento de custeio em um único bloco, mantendo-se grupos de ações dentro do Bloco de Custeio. Esses grupos de ações deverão refletir a vinculação, ao final de cada exercício, do que foi definido em cada programa de trabalho do Orçamento Geral da União e que deu origem ao repasse do recurso, bem como o estabelecido no Plano de Saúde e na Programação Anual de Saúde dos entes subnacionais. No entanto, por ser recente, os reflexos dessa flexibilidade na aplicação, conforme o plano local e eventos, só poderá ser verificado após um período de aplicação e experiência.

\section{MATERIAL E MÉTODO}

A área de estudo abrange Montes Claros que é a cidade-polo na região norte de Minas Gerais e oferece os serviços ambulatoriais e hospitalares de alta complexidade. Conforme o Plano Diretor de Regionalização da Saúde de Minas Gerais (PDR/MG), a RAS Norte de Minas abrange 86 municípios. Estes estão divididos em nove regiões de saúde, com uma população total de aproximadamente 1.609.862 habitantes (SILVEIRA, 2013).

Assim, a pesquisa realizada caracteriza-se como descritiva, em fontes secundárias bibliográficas e de dados oficiais disponibilizados pela Secretaria Municipal de Saúde de Montes Claros, setor contábilfinanceiro. Além dessa fonte, foram consultados o Instituto Brasileiro de Geografia e Estatística (IBGE); o Ministério da Saúde/Secretaria de Atenção à Saúde (SAS)/Departamento de Atenção Básica (DAB).

Neste trabalho, o horizonte temporal situa-se entre 2005 a 2016 que corresponde a três últimas gestões municipais. Foram consideradas variáveis relacionadas a: percentual do orçamento municipal aplicado em saúde; despesas por subfunção; taxa de cobertura do Programa de Saúde da Família.

A consulta ao Sistema de Informações sobre Orçamento Público em Saúde (Siops) foi obtida diretamente na Secretaria Municipal de Saúde de Montes Claros/MG, bem como informações sobre a gestão 3 e não cumprimento da pactuação.

Os dados foram atualizados pela inflação a partir da aplicação do Índice de Preços ao Consumidor Amplo (IPC-A) obtido junto ao Instituto Brasileiro de Geografia e Estatística (IBGE). No que se refere ao processo de atualização, foram utilizados os números-índices referentes à inflação acumulada entre o ano de 2005 (início da série analisada) e o ano a ser atualizado. O número-índice utilizado foi o de junho de cada ano.

\section{APRESENTAÇÃO DOS DADOS E DISCUSSÃO DOS RESULTADOS}

É oportuno iniciar essa seção com a apresentação do cenário de como se dá a confluência do período da gestão do município e do estado, já que o objeto de estudo abrange momentos distintos. O período analisado, 2005 a 2016 contempla 3 gestões municipais em Montes Claros/MG, 2005 a 2008 (gestão 1); 2009 a 2012 (gestão 2); e 2013 a 2016 (gestão 3). Cada entrada do gestor municipal coincide com o terceiro ano de mandato do governador e o término da gestão municipal coincide com o segundo ano do mandato do governador seguinte. Assim, uma administração municipal convive metade de seu mandato com um governador e outra metade com outro, a exceção se dá em casos de reeleição.

Após análise do orçamento Municipal em relação ao orçamento da Saúde, pode-se constatar que a saúde apresenta um comportamento crescente, mesmo que em 2012 as receitas previstas tenham uma diminuição significativa de recursos. Comparando-se as gestões, as despesas em saúde demonstram 
queda a partir de 2015 a 2016, conforme o gráfico 1.

GRÁFICO 1: Orçamento Geral X Orçamento em Saúde X Despesas em Saúde

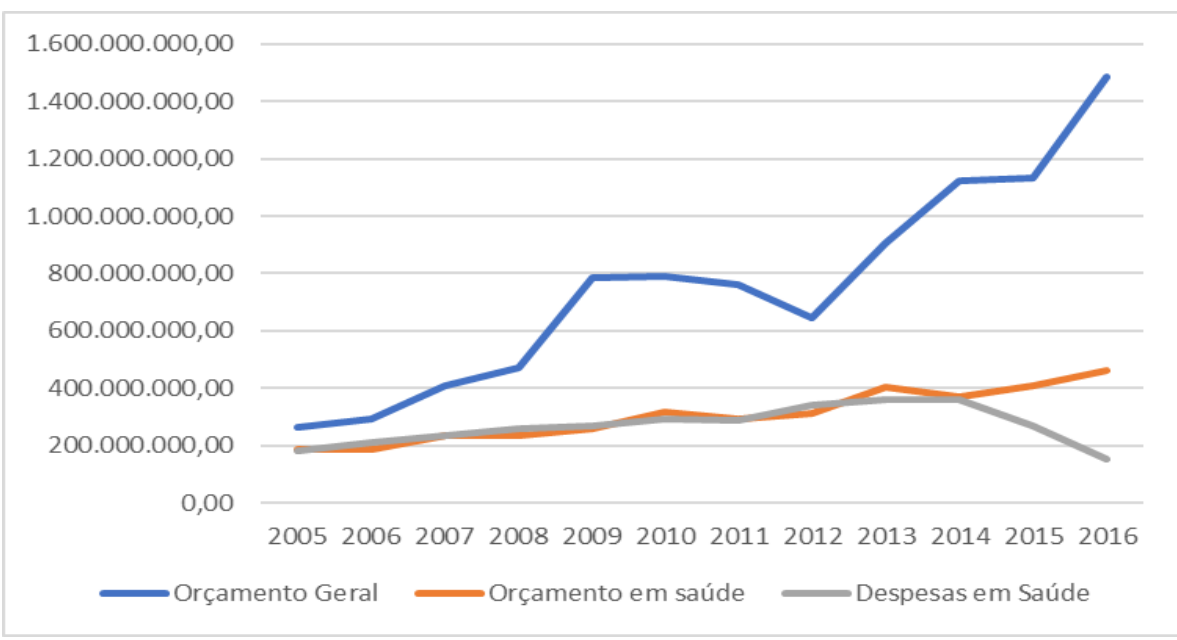

Fonte: SIOPS/Secretaria Municipal de Saúde de Montes Claros/MG, 2018

Sendo uma das propostas deste artigo analisar as gestões municipais e seu comportamento, tem-se que na primeira gestão (2005 a 2008) e na segunda (2009 a 2012) a média de recursos constitucionais aplicados em saúde foi mais de $17 \%$. Ressalta-se, além disso, que a terceira gestão é a que mais aplicou em saúde conforme pode-se observar no QUADRO 1.

Nesse contexto, em termos de percentual constitucional (EMENDA 29) do orçamento municipal aplicado em saúde, percebe-se uma variação entre os anos estudados onde a menor aplicação se apresenta no ano de 2011 com 15,21\% e a maior aplicação em 2015 com 21,29\%, visualizados no GRÁF.2.

QUADRO 1: Média de recursos constitucionais aplicados por gestão no período 2005 a 2016

\begin{tabular}{|l|c|c|c|}
\hline & Gestão 1 & Gestão 2 & Gestão 3 \\
\hline $\begin{array}{l}\text { Média } \\
\text { do período }\end{array}$ & $17,90 \%$ & $17,78 \%$ & $19,73 \%$ \\
\hline
\end{tabular}

Fonte: SIOPS/Secretaria Municipal de Saúde de Montes Claros/MG, 2018

GRÁFICO 2: Aplicação constitucional na saúde (emenda 29)

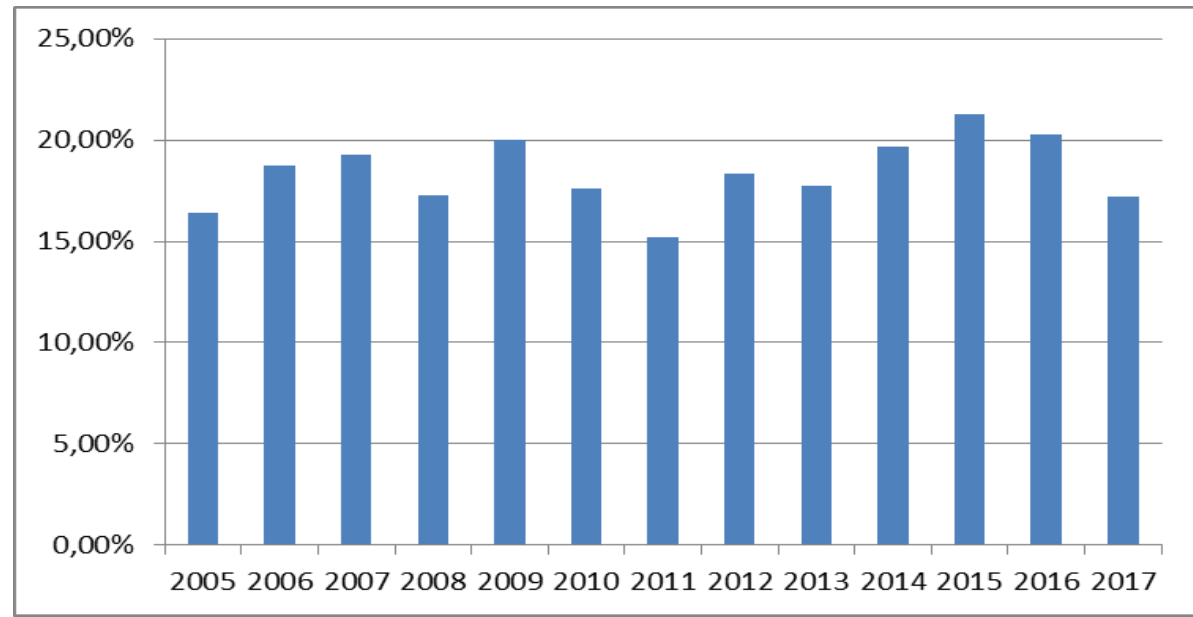

Fonte: SIOPS/Secretaria Municipal de Saúde de Montes Claros/MG, 2018 
As variações que caracterizam cada gestão podem ser detectadas, por exemplo, pela perda da gestão plena no período de 2013 a 2016 que implicou em 7,71\% de acréscimo médio da gestão (3) em relação à gestão (2) no que se refere à atenção básica. É que durante essa gestão (3) o município perdeu a gestão plena consolidada na Portaria do Ministério da Saúde nº 384, de 04 de abril de 2003, e apenas por isso os gastos com a subfunção ambulatorial /hospitalar passou a ser feita pelo estado. O município desonerado investiu em 2016 em atenção básica 59,40\% com uma média de 26,81\% no mandato em contraposição aos 19,10\% dos quatro anos da gestão anterior. A não aplicação do município na média e alta complexidade apenas significa que o Estado assumiu os compromissos pactuados e não necessariamente uma mudança do modelo de saúde adotado pela gestão 3, ou seja, aproximadamente $70 \%$ em média das despesas tem foco na média e alta complexidade, conforme gráfico 3.

GRÁFICO 3: Despesa média em saúde

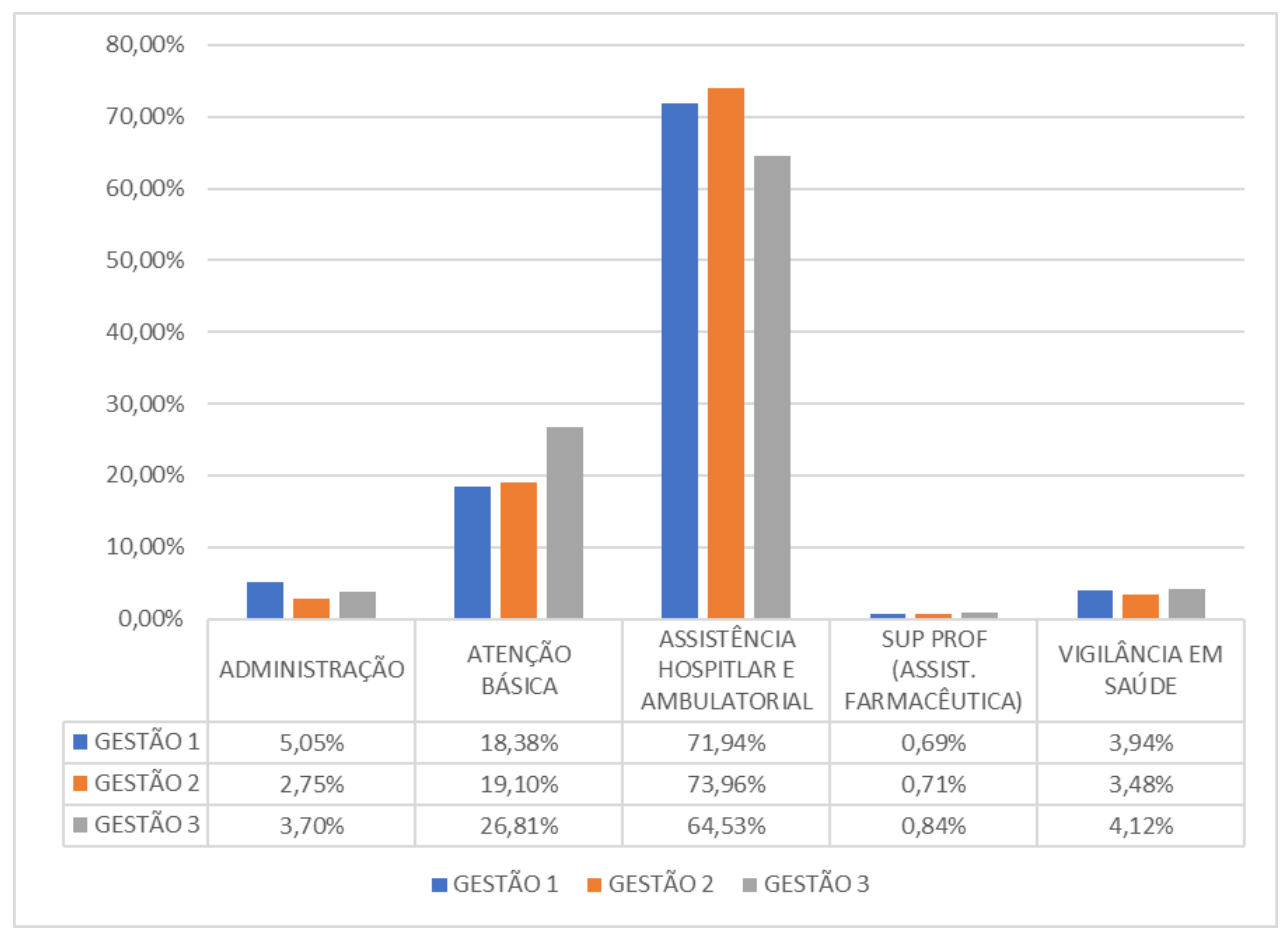

Fonte: SIOPS/Secretaria Municipal de Saúde de Montes Claros/MG, 2018

Ainda com base nos dados do gráfico 3, na gestão 2, o percentual médio de alocação em vigilância em saúde foi ligeiramente inferior à gestão anterior (quase -0,39\%) bem como na subfunção administração $(-2,30 \%)$. Essa última subfunção, também na gestão 3, corresponde a $-1,35 \%$ em relação a gestão 1 .

O gráfico 4 destaca a despesa mais representativa em todas as gestões que é a assistência hospitalar e ambulatorial. Conforme mencionado, em 2015 o município perdeu a gestão plena ${ }^{3}$ visualizando-se esse impacto no município notadamente em 2016, mas mantendo-se outras despesas conforme anos anteriores.

\footnotetext{
${ }^{3}$ Gestão Plena do Sistema Municipal - Transferência, regular e automática, dos recursos referentes ao valor per capita definido para o conjunto mínimo dos procedimentos do M1, após qualificação da microrregião na qual está inserido, para sua própria população e caso seja sede de módulo assistencial, para a sua própria população e população dos municípios abrangidos. - Receber, diretamente no Fundo Municipal de Saúde, o montante total de recursos federais correspondente ao limite financeiro programado para o município, compreendendo a parcela destinada ao atendimento da população própria e aquela destinada ao atendimento à população referenciada, condicionado ao cumprimento efetivo do Termo de Compromisso para Garantia de Acesso firmado
} 
GRÁFICO 4: Subfunção assistência hospitalar e ambulatorial em relação ao total de despesas em saúde

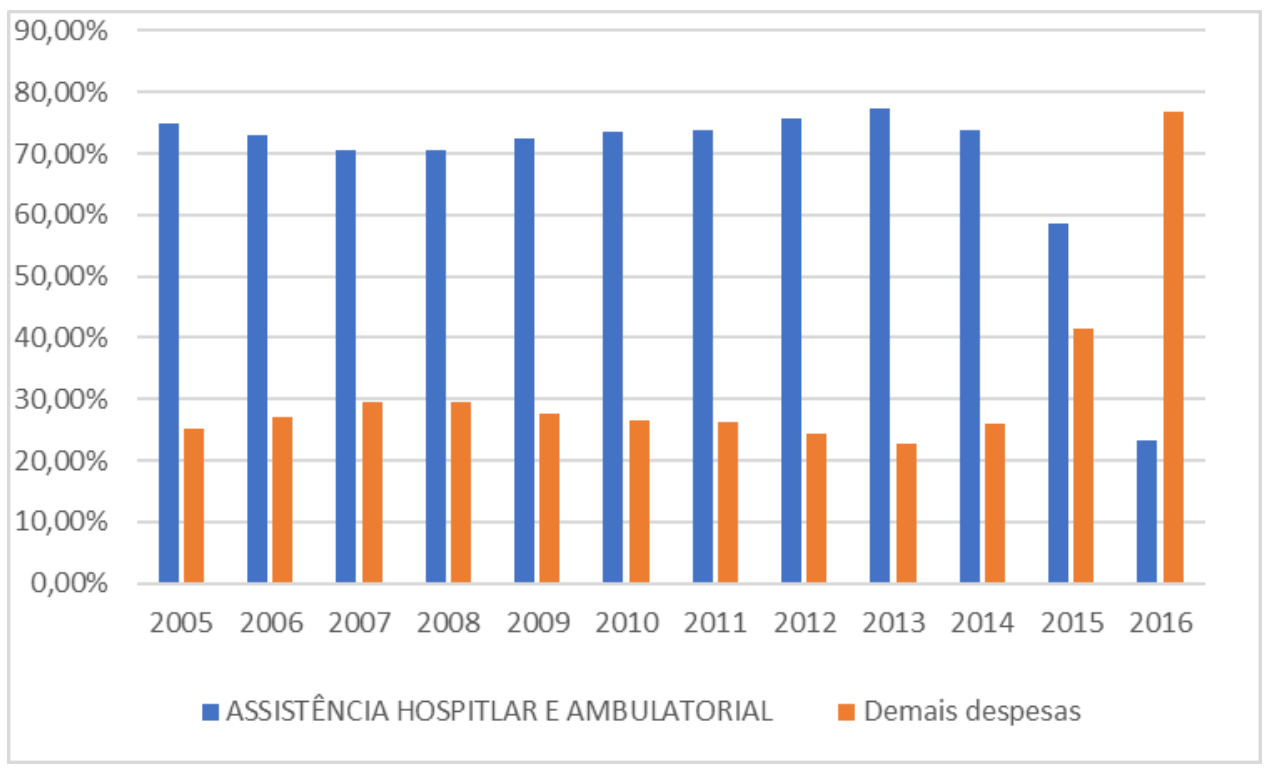

Fonte: SIOPS/Secretaria Municipal de Saúde de Montes Claros/MG, 2018

Na subfunção atenção básica ${ }^{4}$, que visa promoção e prevenção da saúde e é viabilizada pelo programa Estratégia de Saúde da Família, merece destaque a taxa de cobertura da equipe ESF. Desde 2004 é composta por um médico, um enfermeiro, um auxiliar de enfermagem e pelo menos quatro agentes comunitários de saúde, além de profissionais de saúde bucal. As equipes de saúde da família atuam em áreas geográficas definidas e com populações adstritas, contendo até 4000 pessoas para cada equipe, sendo 3000 a média recomendada, podendo ainda esse número ser menor de acordo com o risco e a vulnerabilidade social da população coberta.

Compreendendo o período de 2005 a 2008, a taxa média de cobertura ficou em 36,03\%, ou seja, o município de Montes Claros necessitava de 113 equipes e a administração ofertou 41. No primeiro ano da mesma gestão, com 33 equipes implantadas, levou-se ao resultado de uma cobertura de $30,51 \%$. Nos anos seguintes, 2006, 2007 e 2008, as taxas se posicionaram em 29,76\%, 41,25\% e 42,11\%, respectivamente. Nesse estudo considerou-se como divisor o número de 3000 habitantes.

QUADRO 3: Situação de ESF na gestão municipal de Montes Claros 2005-2008

\begin{tabular}{|l|r|r|r|r|r|}
\hline INDICADOR ANO & \multicolumn{1}{c|}{$\mathbf{2 0 0 5}$} & \multicolumn{1}{c|}{$\mathbf{2 0 0 6}$} & \multicolumn{1}{c|}{$\mathbf{2 0 0 7}$} & \multicolumn{1}{c|}{$\mathbf{2 0 0 8}$} & \multicolumn{1}{c|}{ MÉDIA } \\
\hline População & 324.471 & 342.708 & 349.113 & 349.113 & 341.351 \\
\hline Necessidade de ESF & 108,16 & 114,24 & 116,37 & 116,37 & 113,78 \\
\hline ESF Implantados & 33 & 34 & 48 & 49 & 41 \\
\hline Taxa de cobertura & $30,51 \%$ & $29,76 \%$ & $41,25 \%$ & $42,11 \%$ & $36,03 \%$ \\
\hline
\end{tabular}

Fonte: MS/SAS/DAB e IBGE.

\footnotetext{
${ }^{4}$ A Atenção Básica é um conjunto de ações, de caráter individual e coletivo, situadas no primeiro nível de atenção dos sistemas de saúde, voltadas para a promoção da saúde, a prevenção de agravos, tratamento e a reabilitação (PNAB, 2006)
} 
No segundo período analisado, 2009/2012, a taxa de cobertura média do quadriênio apresentou um resultado de 51,52\%. Um aumento em comparação à administração anterior. Inicia-se com uma cobertura de 48,55\% e entrega ao final de 2012 uma 57,36\%. Nos anos de 2010 e 2011, respectivamente, as taxas ficaram em $48,71 \%$ e $51,39 \%$, respectivamente. Assim como a edilidade anterior, a edilidade presente não conseguiu atender à totalidade da necessidade, embora houvesse um crescimento de $42 \% \mathrm{em}$ termos percentuais, saindo de uma cobertura média de 36,03\% do período anterior para 51,52\% segunda gestão. A necessidade era de 121 equipes e o administrador disponibilizou 62.

QUADRO 4: Situação de ESF na gestão municipal de Montes Claros 2009-2012

\begin{tabular}{|l|r|r|r|r|r|}
\hline INDICADOR ANO & \multicolumn{1}{c|}{$\mathbf{2 0 0 9}$} & \multicolumn{1}{c|}{$\mathbf{2 0 1 0}$} & \multicolumn{1}{c|}{$\mathbf{2 0 1 1}$} & \multicolumn{1}{c|}{$\mathbf{2 0 1 2}$} & \multicolumn{1}{c|}{ MÉDIA } \\
\hline População & 358.393 & 363.349 & 361.915 & 366.135 & 362.448 \\
\hline Necessidade de ESF & 119,46 & 121,12 & 120,64 & 122,05 & 120,82 \\
\hline ESF Implantados & 58 & 59 & 62 & 70 & 62 \\
\hline Taxa de cobertura & $48,55 \%$ & $48,71 \%$ & $51,39 \%$ & $57,36 \%$ & $51,52 \%$ \\
\hline
\end{tabular}

Fonte: MS/SAS/DAB e IBGE.

A terceira gestão, 2013/2016, entrega ao final do seu primeiro ano um percentual de cobertura de $61,59 \%$ e finaliza o ciclo com $101,29 \%$. A taxa de cobertura média no mandato foi de $87,11 \%$ sendo 108 ESF implantados de um total de 123 necessários, considerando 3000 habitantes por ESF. Aumento de $74 \%$ de ESF implantados em relação à administração anterior e de $163 \%$ relativamente à primeira administração municipal estudada (QUADRO 5).

QUADRO 5: Situação de ESF na gestão municipal de Montes Claros 2013-2016

\begin{tabular}{|l|r|r|r|r|r|}
\hline INDICADOR ANO & \multicolumn{1}{c|}{$\mathbf{2 0 1 3}$} & \multicolumn{1}{c|}{$\mathbf{2 0 1 4}$} & \multicolumn{1}{c|}{$\mathbf{2 0 1 5}$} & \multicolumn{1}{c|}{$\mathbf{2 0 1 6}$} & \multicolumn{1}{c|}{ MÉDIA } \\
\hline População & 370.216 & 370.216 & 370.216 & 370.216 & 370.216 \\
\hline Necessidade de ESF & 123,41 & 123,41 & 123,41 & 123,41 & 123,41 \\
\hline ESF Implantados & 76 & 100 & 129 & 125 & 108 \\
\hline Taxa de cobertura & $61,59 \%$ & $81,03 \%$ & $104,53 \%$ & $101,29 \%$ & $87,11 \%$ \\
\hline
\end{tabular}

Fonte: MS/SAS/DAB e IBGE.

Nos dois últimos anos de mandato, 2015 e 2016, quando o estado assumiu as despesas de média e alta complexidade, o município não repactuou com base em indicadores epidemiológicos e de demanda, apenas transferiu responsabilidades. Portanto, observa-se no gráfico 5 a ênfase nas equipes de Estratégia de Saúde da Família e aumento da cobertura. 
GRÁFICO 5: Subfunção atenção básica em relação ao total de despesas

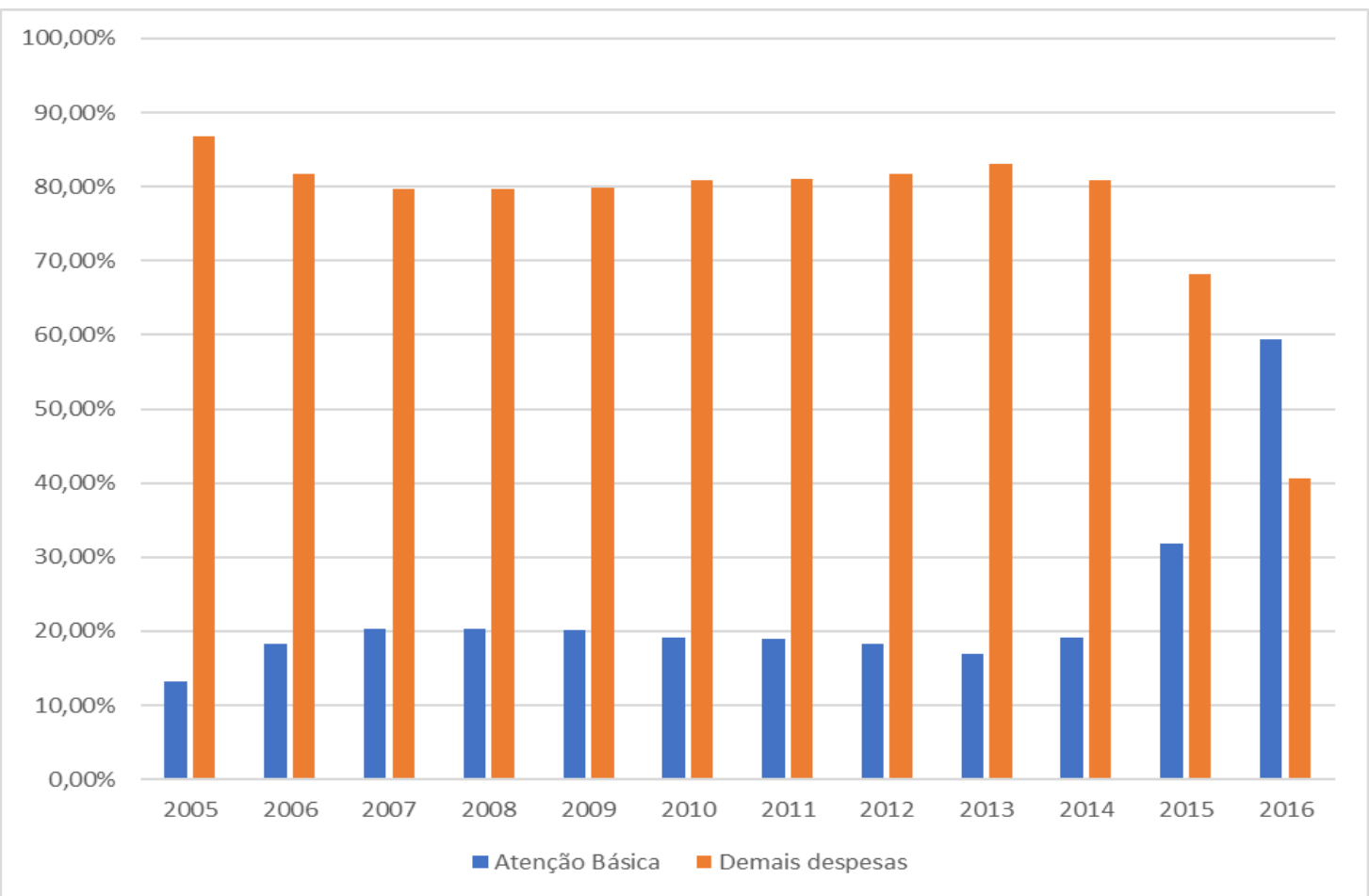

Fonte: SIOPS/Secretaria Municipal de Saúde de Montes Claros/MG, 2018

A taxa de cobertura do ESF e certa mudança do modelo de saúde adotado pelo município, mesmo que temporário, estão refletidos também no GRÁF. 5, ao se comparar as diferentes gestões.

Em todas as gestões estudadas a subfunção com menor participação (menos de 1\%) é Suporte profilático e terapêutico (Assistência farmacêutica) ${ }^{5}$. A vigilância em saúde, coerentemente aos eventos apresentados, tem maior percentual de gastos na terceira gestão em relação a gestões anteriores (4\%).

\section{CONSIDERAÇÕES FINAIS}

De uma forma geral, os indicadores de gastos com saúde em sua série histórica indicam que o município pólo de uma região, Montes Claros, gasta principalmente com a média e alta complexidade, ou seja, com assistência hospitalar. Independente do orçamento maior ou menor observa-se que os gastos com saúde são crescentes e constantes. No entanto, a cobertura da população pelo Programa Saúde da Família ainda não acontece em sua plenitude, o que se supõe minimizaria os gastos com alta complexidade.

Por outro lado, uma vez pactuado, independente de mudanças nos indicadores epidemiológicos, supõe-se que o modelo se mantém, sobretudo em municípios polos. Este recebe pacientes de vasta região com diferentes infraestruturas de saúde e de trabalho de prevenção e promoção. Assim, existe uma pactuação já consolidada e supostamente difícil de reverter.

O trabalho deve ter continuidade para buscar em fontes primárias, junto ao gestor municipal, as

\footnotetext{
${ }^{5}$ Grupo de atividades relacionadas com o medicamento, destinadas a apoiar as ações de saúde demandadas por uma comunidade. Envolve o abastecimento de medicamentos em todas e em cada uma de suas etapas constitutivas, a conservação e o controle de qualidade, a segurança e a eficácia terapêutica dos medicamentos, o acompanhamento e a avaliação da utilização, a obtenção e a difusão de informação sobre medicamentos e a educação permanente dos profissionais de saúde, do paciente e da comunidade para assegurar o uso racional de medicamentos. (BRASIL, 2002a, p.34).
} 
ações e eventos que caracterizaram cada gestão, bem como o comportamento dos indicadores epidemiológicos locais.

\section{REFERÊNCIAS}

BRASIL. GOVERNO FEDERAL. Lei no 8.080: Sistema Único de Saúde (SUS), Brasília, 19/09/1990.

BRASIL. GOVERNO FEDERAL. Lei n. ${ }^{\circ}$ 4320/64, Brasília, 17 de março de 1964.

BRASIL. GOVERNO FEDERAL. LEI No 8.142, Brasília, 28 de dezembro de 1990.

BRASIL. GOVERNO FEDERAL. Lei Complementar n. ${ }^{\mathbf{1}}$ 101/2000, Brasília, 4 de maio de 2000.

BRASIL. MINISTÉRIO DA SAÚDE. PORTARIA No 699/GM. Brasília, 30 de março de 2006.

BRASIL. MINISTÉRIO DO PLANEJAMENTO, ORCAMENTO E GESTÃO. PORTARIA MOG no 42, Brasília, 14 de abril de 1999.

BRASIL. MINISTÉRIO DA SAÚDE. Portaria n 648/GM . Brasília, 28 de março de 2006.

COELHO, V.S.P; SZABZON,F.; DIAS, M.F. Política municipal e acesso a serviços de saúde São Paulo 2001-2012, quando as periferias ganharam mais que o centro.Novos estud. - CEBRAP no.100 São Paulo Nov. 2014.

CONSElHO NACIONAL DE SECRETÁRIOS DE SAÚDE - CONASS. Coleção Progestores - Para entender a gestão do SUS, Brasília, 2007. Disponível em: <www.conass.org.br>. Acesso em: 22 ago.2018.

COUTTOLENC BF;ZUCCHI P. Saúde \& cidadania para gestores municipais de serviços de saúde [CD-ROM]. Livro 10. $2^{\mathrm{a}}$ ed. Versão 2.0. Brasília: Ministério da Saúde, 2002.

BRASIL. GOVERNO FEDERAL. Emenda Constitucional no 29 (EC 29). Brasília, 14 de abril de 2012.

KRUSCHE, C.; CERETTA, P.S. O orçamento como instrumento de planejamento para uma gestão publica eficaz. Monografia de Pós-graduação em gestão pública municipal. São Paulo/Santa Maria, Universidade Federal de Santa Maria (UFSM), 2012.

MENDES, Eugênio Vilaçã. A modelagem das redes de atenção à saúde. Belo Horizonte: Secretaria de Estado da Saúde de Minas Gerais, 2002.

MENDES, EV. Revisão bibliográfica sobre redes de atenção à saúde. Belo Horizonte: Secretaria de Estado da Saúde de Minas Gerais; 2007.

SILVEIRA, Y. M. S. C. A regionalização da saúde no estado de Minas Gerais e o papel de Montes Claros nesse contexto. In: RAMIRES, J. C. L. (Org.). Dinâmica socioespacial e saúde coletiva em cidades de Minas Gerais. Rio de Janeiro: Letra Capital, 2013. p. 165-191.

WEILLER, J.A. B.; MENDES, Á.N. O Orçamento por Desempenho como ferramenta para gestão e avaliação da política de saúde no município de São Bernardo do Campo, no período 2006 a 2012. In: Saúde debate 40 (110) Jul-Sep 2016 • https://doi.org/10.1590/0103-1104201611003 . São Paulo: Universidade de São Paulo (USP), Faculdade de Saúde Pública - São Paulo (SP), 2016.

XXVII CONGRESSO DE SECRETÁRIOS MUNICIPAIS DE SAÚDE DO ESTADO DE SÃO PAULO. COSEMS/SP. Desafios da Gestão Municipal do SUS na articulação com o estado e a União, março de 2013. Disponível em: <www.cosemssp.org.br/congresso2013>. Acesso em: 22 ago. 2018. 


\section{APÊNDICE A}

\section{RECEITAS (BASE DE CÁLCULO APLICAÇÃO EM SAÚDE - MUNICÍPIO) GESTÃO 1}

\begin{tabular}{|c|c|c|c|c|}
\hline ANO/INDICADOR & 2005 & 2006 & 2007 & 2008 \\
\hline ITR & 0,00 & 0,00 & 0,00 & 0,00 \\
\hline IPTU & $11.843 .511,67$ & $13.787 .560,46$ & $13.213 .118,12$ & 14.342.091,11 \\
\hline IRRF & $4.439 .153,55$ & $5.755 .554,77$ & $6.739 .477,84$ & $7.974 .344,93$ \\
\hline ITBI & $3.522 .172,77$ & $4.126 .161,08$ & $4.409 .102,10$ & $6.135 .807,91$ \\
\hline ISS & $23.531 .810,28$ & 24.125.339,84 & $28.368 .908,24$ & $30.880 .380,01$ \\
\hline $\begin{array}{c}\text { Multas e Juros de } \\
\text { Impostos } \\
\end{array}$ & $1.143 .490,26$ & $1.606 .102,36$ & $1.251 .151,87$ & $663.231,77$ \\
\hline $\begin{array}{c}\text { Multas e Juros Dívida } \\
\text { Ativa } \\
\end{array}$ & $539.931,01$ & $1.046 .410,23$ & $1.224 .014,31$ & $2.197 .602,52$ \\
\hline $\begin{array}{c}\text { Dívida Ativa de } \\
\text { Impostos } \\
\end{array}$ & $3.918 .487,72$ & $8.555 .122,65$ & $10.993 .797,48$ & $9.941 .495,86$ \\
\hline Cota-parte FPM & $53.897 .630,70$ & $57.685 .216,06$ & $63.830 .965,44$ & $76.028 .761,49$ \\
\hline Cota-parte ITR & $100.739,01$ & $115.338,52$ & $245.033,15$ & $188.188,08$ \\
\hline $\begin{array}{l}\text { Lei Compl. } N^{\circ} .87 / 96 \text { - } \\
\text { Lei Kandir }\end{array}$ & $2.257 .879,66$ & $1.113 .112,95$ & $956.978,43$ & $1.020 .818,62$ \\
\hline Cota-parte do ICMS & $80.699 .555,34$ & $75.283 .208,62$ & $80.308 .757,06$ & $94.867 .123,87$ \\
\hline Cota-parte IPVA & $18.112 .119,97$ & $21.408 .492,41$ & $24.864 .551,37$ & $27.111 .165,35$ \\
\hline Cota-parte do IPI & $1.725 .923,41$ & $1.319 .320,68$ & $2.724 .893,35$ & $1.802 .521,56$ \\
\hline TOTAL & $\mathrm{R} \$ 205.732 .405,35$ & $\mathrm{R} \$ 115.034 .995,29$ & R\$132.097.510,72 & $\mathrm{R} \$ 160.036 .868,13$ \\
\hline VARIAÇÃO \% & & $9,18 \%$ & $14,83 \%$ & $21,15 \%$ \\
\hline
\end{tabular}

\section{GESTÃO 2}

\begin{tabular}{|c|r|r|r|r|}
\hline ANO/INDICADOR & \multicolumn{1}{|c|}{$\mathbf{2 0 0 9}$} & \multicolumn{1}{|c|}{$\mathbf{2 0 1 0}$} & \multicolumn{1}{c|}{$\mathbf{2 0 1 1}$} & \multicolumn{1}{c|}{$\mathbf{2 0 1 2}$} \\
\hline ITR & 0,00 & 0,00 & 0,00 & 0,00 \\
\hline IPTU & $15.743 .854,12$ & $19.250 .239,30$ & $19.191 .842,12$ & $18.194 .907,66$ \\
\hline IRRF & $6.707 .537,15$ & $5.477 .756,69$ & $6.647 .750,56$ & $8.681 .931,56$ \\
\hline ITBI & $6.932 .586,85$ & $11.395 .152,44$ & $14.350 .568,16$ & $15.802 .817,80$ \\
\hline ISS & $32.771 .601,92$ & $34.138 .825,25$ & $39.160 .373,31$ & $41.795 .541,66$ \\
\hline $\begin{array}{c}\text { Multas e Juros de } \\
\text { Impostos }\end{array}$ & $1.430 .129,87$ & $1.588 .944,04$ & $1.598 .320,09$ & $950.170,19$ \\
\hline $\begin{array}{c}\text { Multas e Juros Dívida } \\
\text { Ativa }\end{array}$ & $3.335 .921,43$ & $4.676 .198,85$ & $2.877 .590,30$ & $663.132,47$ \\
\hline $\begin{array}{c}\text { Dívida Ativa de } \\
\text { Impostos }\end{array}$ & $5.746 .244,03$ & $9.216 .827,55$ & $5.743 .898,22$ & $1.968 .411,43$ \\
\hline Cota-parte FPM & $67.192 .296,07$ & $69.996 .569,00$ & $79.890 .310,59$ & $78.323 .745,95$ \\
\hline Cota-parte ITR & $140.943,59$ & $140.290,55$ & $142.160,27$ & $122.202,84$ \\
\hline $\begin{array}{c}\text { Lei Compl. N } \text {. 87/96 - } \\
\text { Lei Kandir }\end{array}$ & $902.871,94$ & $807.576,44$ & $851.187,46$ & $822.658,17$ \\
\hline Cota-parte do ICMS & $80.345 .258,22$ & $87.973 .830,96$ & $99.166 .606,82$ & $104.589 .089,49$ \\
\hline Cota-parte IPVA & $29.667 .455,53$ & $31.864 .071,13$ & $34.551 .363,86$ & $39.359 .481,46$ \\
\hline Cota-parte do IPI & $1.233 .271,13$ & $1.576 .166,38$ & $1.604 .572,14$ & $1.996 .204,28$ \\
\hline & & & & \\
\hline TOTAL & $\mathbf{R} \mathbf{\$ 1 5 4 . 8 2 4 . 5 8 1 , 7 2}$ & $\mathbf{R \$ 1 7 9 . 0 2 6 . 5 0 5 , 3 3}$ & $\mathbf{R \$ 2 1 0 . 0 5 4 . 5 9 6 , 7 6}$ & $\mathbf{R \$ 2 2 5 . 7 8 0 . 5 7 9 , 6 6}$ \\
\hline VARIAÇÃO \% & $-3,26 \%$ & $15,63 \%$ & $17,33 \%$ & $7,49 \%$ \\
\hline
\end{tabular}


GESTÃO 3

\begin{tabular}{|c|r|r|r|r|}
\hline ANO/INDICADOR & \multicolumn{1}{|c|}{$\mathbf{2 0 1 3}$} & \multicolumn{1}{|c|}{$\mathbf{2 0 1 4}$} & \multicolumn{1}{c|}{$\mathbf{2 0 1 5}$} & \multicolumn{1}{c|}{$\mathbf{2 0 1 6}$} \\
\hline ITR & 0,00 & 0,00 & 0,00 & 0,00 \\
\hline IPTU & $24.130 .077,88$ & $23.356 .577,92$ & $22.235 .720,52$ & $25.627 .370,72$ \\
\hline IRRF & $7.993 .188,80$ & $10.551 .743,21$ & $3.729 .583,10$ & $9.628 .688,68$ \\
\hline ITBI & $18.418 .499,80$ & $21.423 .818,87$ & $19.168 .103,29$ & $18.084 .424,92$ \\
\hline ISS & $52.125 .173,65$ & $56.505 .581,86$ & $55.047 .920,92$ & $51.392 .476,89$ \\
\hline $\begin{array}{c}\text { Multas e Juros de } \\
\text { Impostos }\end{array}$ & $2.330 .002,06$ & $1.724 .867,09$ & $1.303 .355,35$ & $1.958 .766,71$ \\
\hline $\begin{array}{c}\text { Multas e Juros } \\
\text { Dívida Ativa }\end{array}$ & $1.857 .702,25$ & $2.681 .102,12$ & $2.427 .534,34$ & $2.501 .083,90$ \\
\hline $\begin{array}{c}\text { Dívida Ativa de } \\
\text { Impostos }\end{array}$ & $6.027 .707,45$ & $9.062 .805,95$ & $7.235 .282,13$ & $7.255 .896,68$ \\
\hline Cota-parte FPM & $77.903 .288,19$ & $78.373 .160,00$ & $76.577 .510,91$ & $76.226 .619,06$ \\
\hline Cota-parte ITR & $139.234,08$ & $117.521,61$ & $109.804,29$ & $135.575,10$ \\
\hline $\begin{array}{c}\text { Lei Compl. } \mathbf{N}^{\mathbf{0} .87 / 96} \\
\text { - Lei Kandir }\end{array}$ & $749.228,90$ & $710.444,19$ & $686.790,40$ & $680.442,70$ \\
\hline Cota-parte do ICMS & $108.662 .991,70$ & $108.656 .527,07$ & $103.320 .192,44$ & $111.231 .179,92$ \\
\hline Cota-parte IPVA & $40.622 .680,13$ & $43.622 .432,40$ & $44.672 .143,93$ & $45.758 .425,44$ \\
\hline Cota-parte do IPI & $1.552 .623,47$ & $1.983 .540,20$ & $1.876 .984,47$ & $1.381 .919,96$ \\
\hline & & & & \\
\hline TOTAL & $\mathbf{R} \$ \mathbf{2 6 3 . 3 8 5 . 2 5 6 , 0 6}$ & $\mathbf{R \$ 2 9 3 . 8 8 4 . 0 2 3 , 7 0}$ & $\mathbf{R \$ 3 0 1 . 8 4 5 . 0 6 7 , 4 0}$ & $\mathbf{R \$ 3 4 1 . 6 1 9 . 2 2 3 , 5 8}$ \\
\hline VARIAÇÃO \% & $16,66 \%$ & $11,58 \%$ & $2,71 \%$ & $13,18 \%$ \\
\hline
\end{tabular}

\begin{tabular}{|c|c|c|c|c|}
\hline \multicolumn{5}{|c|}{ GESTÃO 1 } \\
\hline ANO/INDICADOR & $\mathbf{2 0 1 3}$ & $\mathbf{2 0 1 4}$ & $\mathbf{2 0 1 5}$ & $\mathbf{2 0 1 6}$ \\
\hline ANO/INDICADOR & $\mathbf{2 0 0 5}$ & $\mathbf{2 0 0 6}$ & $\mathbf{2 0 0 7}$ & $\mathbf{2 0 0 8}$ \\
\hline ADMINISTRAÇÃO & $8,53 \%$ & $4,61 \%$ & $3,55 \%$ & $4,34 \%$ \\
\hline ATENÇÃO BÁSICA & $13,21 \%$ & $18,37 \%$ & $20,24 \%$ & $20,29 \%$ \\
\hline ASSISTÊNCIA & & & & $70,45 \%$ \\
HOSPITLAR E & $74,91 \%$ & $72,92 \%$ & $70,44 \%$ & \\
AMBULATORIAL & & & & \\
\hline
\end{tabular}

\section{APLICAÇÃO CONSTITUCIONAL NA SAÚDE (EMENDA 29)}

\begin{tabular}{|c|c|c|c|c|}
\hline ANO/INDICADOR & $\mathbf{2 0 0 5}$ & $\mathbf{2 0 0 6}$ & $\mathbf{2 0 0 7}$ & $\mathbf{2 0 0 8}$ \\
\hline APLICACAO \% GESTÃO 1 & $16,41 \%$ & $18,72 \%$ & $19,26 \%$ & $17,24 \%$ \\
\hline ANO/INDICADOR & 2009 & 2010 & 2011 & 2012 \\
\hline APLICACAO \% GESTÃO 2 & $20,00 \%$ & $17,58 \%$ & $15,21 \%$ & $18,34 \%$ \\
\hline ANO/INDICADOR & 2013 & 2014 & 2015 & 2016 \\
\hline APLICACAO \% GESTÃO 3 & $17,72 \%$ & $19,64 \%$ & $21,29 \%$ & $20,30 \%$ \\
\hline
\end{tabular}


DESPESA TOTAL DA SAÚDE POR SUBFUNÇÃO em percentual(TODAS AS FONTES)

\begin{tabular}{|c|c|c|c|c|}
\hline \multicolumn{5}{|c|}{ GESTÃO 1} \\
\hline ANO/INDICADOR & 2005 & 2006 & 2007 & 2008 \\
\hline ADMINISTRAÇÃO & $8,53 \%$ & $4,61 \%$ & $3,55 \%$ & $4,34 \%$ \\
\hline ATENÇÃO BÁSICA & $13,21 \%$ & $18,37 \%$ & $20,24 \%$ & $20,29 \%$ \\
\hline $\begin{array}{c}\text { ASSISTÊNCIA } \\
\text { HOSPITLAR E } \\
\text { AMBULATORIAL }\end{array}$ & $74,91 \%$ & $72,92 \%$ & $70,44 \%$ & $70,45 \%$ \\
\hline $\begin{array}{c}\text { SUPORTE } \\
\text { PROFILÁTICO E } \\
\text { TERAPÊUTICO } \\
\text { (ASSIST. } \\
\text { FARMACÊUTICA) } \\
\end{array}$ & $0,18 \%$ & $0,41 \%$ & $1,32 \%$ & $0,71 \%$ \\
\hline $\begin{array}{l}\text { VIGILÂNCIA EM } \\
\text { SAÚDE }\end{array}$ & $3,17 \%$ & $3,70 \%$ & $4,45 \%$ & $4,20 \%$ \\
\hline \multicolumn{5}{|c|}{ GESTÃO 2} \\
\hline ANO/INDICADOR & 2009 & 2010 & 2011 & 2012 \\
\hline ADMINISTRAÇÃO & $3,09 \%$ & $3,06 \%$ & $2,82 \%$ & $2,17 \%$ \\
\hline ATENÇÃO BÁSICA & $20,18 \%$ & $19,21 \%$ & $18,93 \%$ & $18,32 \%$ \\
\hline $\begin{array}{c}\text { ASSISTÊNCIA } \\
\text { HOSPITLAR E } \\
\text { AMBULATORIAL }\end{array}$ & $72,40 \%$ & $73,43 \%$ & $73,87 \%$ & $75,73 \%$ \\
\hline $\begin{array}{c}\text { SUPORTE } \\
\text { PROFILÁTICO E } \\
\text { TERAPÊUTICO } \\
\text { (ASSIST. } \\
\text { FARMACÊUTICA) }\end{array}$ & $0,68 \%$ & $0,77 \%$ & $0,81 \%$ & $0,59 \%$ \\
\hline $\begin{array}{l}\text { VIGILÂNCIA EM } \\
\text { SAÚDE }\end{array}$ & $3,65 \%$ & $3,54 \%$ & $3,58 \%$ & $3,20 \%$ \\
\hline \multicolumn{5}{|c|}{ GESTÃO 3} \\
\hline ANO/INDICADOR & 2013 & 2014 & 2015 & 2016 \\
\hline ADMINISTRAÇÃO & $2,59 \%$ & $3,16 \%$ & $3,86 \%$ & $7,30 \%$ \\
\hline ATENÇÃO BÁSICA & $16,91 \%$ & $19,18 \%$ & $31,74 \%$ & $59,40 \%$ \\
\hline $\begin{array}{c}\text { ASSISTÊNCIA } \\
\text { HOSPITLAR E } \\
\text { AMBULATORIAL }\end{array}$ & $77,23 \%$ & $73,90 \%$ & $58,53 \%$ & $23,16 \%$ \\
\hline $\begin{array}{c}\text { SUPORTE } \\
\text { PROFILÁTICO E } \\
\text { TERAPÊUTICO } \\
\text { (ASSIST. } \\
\text { FARMACÊUTICA) }\end{array}$ & $0,60 \%$ & $0,78 \%$ & $0,93 \%$ & $1,37 \%$ \\
\hline $\begin{array}{l}\text { VIGILÂNCIA EM } \\
\text { SAÚDE } \\
\end{array}$ & $2,67 \%$ & $2,99 \%$ & $4,94 \%$ & $8,77 \%$ \\
\hline
\end{tabular}

